\title{
Approximation properties of lowest-order hexahedral Raviart-Thomas finite elements
}

\section{Proprietés d'approximation des éléments finis de Raviart-Thomas hexaédriques d'ordre le plus bas}

\author{
Alfredo Bermúdez ${ }^{\mathrm{a}}$, Pablo Gamallo ${ }^{\mathrm{b}}$, María R. Nogueiras ${ }^{\mathrm{a}}$, Rodolfo Rodríguez ${ }^{\mathrm{c}}$ \\ ${ }^{a}$ Departamento de Matemática Aplicada, Universidade de Santiago de Compostela, 15782 Santiago de Compostela, Spain \\ ${ }^{\mathrm{b}}$ Institute of Sound and Vibration Research, University of Southampton, Highfield Road, Southampton SO17 1BJ, UK \\ ${ }^{\mathrm{c}} \mathrm{GI}^{2} \mathrm{MA}$, Departamento de Ingeniería Matemática, Universidad de Concepción, Chile
}

\begin{abstract}
Basic interpolation results are settled for lowest-order hexahedral Raviart-Thomas finite elements. Convergence in $\mathrm{H}$ (div) is proved for regular families of asymptotically parallelepiped meshes. The need of the asymptotically parallelepiped assumption is demonstrated with a numerical example.

\section{Résumé}

Nous démontrons quelques résultats d'interpolation pour les éléments finis de Raviart-Thomas hexaédriques d'ordre le plus bas. Nous prouvons convergence dans l'espace $\mathrm{H}(\mathrm{div})$ pour des familles régulières de maillages dont les éléments sont, asymptotiquement, des parallélépipèdes. La nécessité de cette hypothèse est montrée numériquement avec un exemple.
\end{abstract}

\section{Version française abrégée}

Le but de cet article est d'étudier les propriétés d'approximation des éléments finis de Raviart-Thomas hexaédriques d'ordre le plus bas. Ces éléments ont été utilisés avec succès dans beaucoup d'applications comme par exemple la vibroacoustique (voir $[5,9,10]$ ).

L'optimalité de l'approximation des fonctions régulières pour des maillages rectangulaires (dans 2D) ou cubiques (dans 3D) a été démontrée dans [14,15] et [13], respectivement. Pour des maillages quadrilatéraux plus généraux, l'optimalité en norme $\mathrm{L}^{2}$ est prouvée dans [15], mais la convergence optimale dans $\mathrm{H}$ (div) n'y est démontrée que pour des familles de maillages dont les éléments soient asymptotiquement des parallélogrammes, plus précisément, formés de quadrilatères tels que la distance de leurs sommets aux sommets d'un parallélogramme soit d'ordre supérieur. Plus récemment, Arnold et al. [1,3] ont démontré qu'en effet l'ordre de convergence peut se détériorer quand ont utilise des éléments finis non standard sur certains maillages quadrilatéraux (donc en 2D), même s'ils sont réguliers. Ces auteurs ont aussi démontré dans [2], la dégradation de la convergence pour des

Email addresses: mabermud@usc.es (Alfredo Bermúdez), pg1@isvr.soton.ac.uk (Pablo Gamallo), marianog@usc.es (María R. Nogueiras), rodolfo@ing-mat . udec. cl (Rodolfo Rodríguez). 
maillages de trapèzes par rapport à des maillages rectangulaires, dans le cas d'espaces d'éléments finis vectoriels définis par la transformée de Piola (dont un cas particulier sont les éléments de Raviart-Thomas).

Dans cet article nous montrons que la convergence dans $\mathrm{H}(\mathrm{div})$ est préservée sur des maillages réguliers formés d'hexaèdres qui soient asymptotiquement des parallélépipèdes. Nous montrons aussi la nécessité de cette hypothèse avec un exemple numérique.

On commence par considérer des maillages d'hexaèdres $K$ qui sont obtenus comme des images du cube unité $\widehat{K}$ par des applications trilinéaires $\boldsymbol{F}_{K}$. On note par $h_{K}$ le diamètre de $K$ et par $\varepsilon_{K}$ une constante qui nous indique combien $K$ est loin d'un parallélépipède. Alors, on démontre des estimations pour les dérivées des termes qui interviennent dans la transformée de Piola; elles sont données dans (1) et le Lemme 2.1. Avec ces estimations on peut démontrer le résultat principal d'approximation ainsi que le corollaire ci-dessous :

Theorem 0.1 Soit $\boldsymbol{u} \in \mathrm{H}(\operatorname{div}, K) \cap \mathrm{H}^{r}(K)^{3}$ avec $0<r \leq 1$. Alors il existe $C>0$, qui ne dépend que de la régularité de $K$ et de $r$, telle que

$$
\left\|\boldsymbol{u}-\boldsymbol{u}_{K}\right\|_{0, K} \leq C\left(\varepsilon_{K} h_{K}^{-1}\|\boldsymbol{u}\|_{0, K}+h_{K}^{r}|\boldsymbol{u}|_{r, K}+h_{K}\|\operatorname{div} \boldsymbol{u}\|_{0, K}\right),
$$

Si, en plus, $\operatorname{div} \boldsymbol{u} \in \mathrm{H}^{s}(K)$ avec $0<s \leq 1$, alors il existe $C>0$, qui ne dépend que de la régularité de $K$ et de $s$, telle que

$$
\left\|\operatorname{div}\left(\boldsymbol{u}-\boldsymbol{u}_{K}\right)\right\|_{0, K} \leq C\left(\varepsilon_{K} h_{K}^{-1}\|\operatorname{div} \boldsymbol{u}\|_{0, K}+h_{K}^{s}|\operatorname{div} \boldsymbol{u}|_{s, K}\right)
$$

Corollary 0.2 Soit $\boldsymbol{u} \in \mathrm{H}^{r}(\Omega)^{3}$ avec $0<r \leq 1$ telle que $\operatorname{div} \boldsymbol{u} \in \mathrm{H}^{s}(\Omega)$ avec $0<s \leq 1$. Soit $\boldsymbol{u}^{\mathrm{R}}$ son interpolant de Raviart-Thomas. Si $\left\{\mathcal{T}_{h}\right\}$ est une famille régulière de maillages du domaine $\Omega$, dont les éléments sont asymptotiquement des parallélépipèdes, alors on a

$$
\left\|\boldsymbol{u}-\boldsymbol{u}^{\mathrm{R}}\right\|_{0, \Omega} \leq C h^{r}\left(\|\boldsymbol{u}\|_{r, \Omega}+\|\operatorname{div} \boldsymbol{u}\|_{0, \Omega}\right) \quad \text { et } \quad\left\|\operatorname{div}\left(\boldsymbol{u}-\boldsymbol{u}^{\mathrm{R}}\right)\right\|_{0, \Omega} \leq C h^{s}\|\operatorname{div} \boldsymbol{u}\|_{s, \Omega} .
$$

Finalement, la nécessité de l'hypothèse ci-dessus sur les maillages est montrée numériquement avec un exemple. Pour cela on utilise les éléments analysés dans cet article afin de calculer les modes propres acoustiques d'une cavité bidimensionnelle, carrée, rigide, à partir d'une formulation en déplacements. On utilise successivement un maillage de trapèzes semblables au trapèze de sommets $(0,0),\left(\frac{1}{2}, 0\right),\left(\frac{1}{2}, \frac{2}{3}\right)$ et $\left(0, \frac{1}{3}\right)$ (voir Fig. 2), qui ne sont pas asymptotiquement des parallélogrammes et un autre uniforme formé par des carrés. Les résultats sont donnés dans les tables 1 et 2 . On constate que la méthode converge dans le deux cas avec un ordre quadratique. Cependant, dans le cas des trapèzes, la méthode converge vers une valeur propre fausse, ce qui ne peut arriver que si $\left\|\boldsymbol{u}-\boldsymbol{u}^{\mathrm{R}}\right\|_{\mathrm{H}(\operatorname{div}, \Omega)}$ ne converge pas vers zéro quand $h$ tends vers zéro.

\section{Introduction}

The aim of this paper is to study the approximation properties of lowest-order hexahedral Raviart-Thomas finite elements. This kind of elements have been introduced by Raviart and Thomas in $[14,15]$ for 2D meshes built on triangles or quadrilaterals and extended by Nédélec in [13] to 3D meshes built on tetrahedra or cubes. These elements have been successfully used in several many applications; for instance, to discretize fluid displacements in fluid-structure vibroacoustic interaction problems (v.g., [5,9,10]). In this type of problems, Raviart-Thomas elements based on hexahedra proved to be much more efficient than those based on tetrahedra (see [7,8]).

Raviart-Thomas elements are $\mathrm{H}$ (div)-conforming. They provide optimal-order approximations in $\mathrm{H}$ (div) of smooth vector fields on 2D shape-regular rectangular meshes ([14]) or 3D cubic meshes ([13]). A deep analysis of these elements in 2D has been made by Thomas in [15], where optimal-order $\mathrm{L}^{2}$-convergence has been proved for general shape-regular quadrilaterals. However, $\mathrm{H}(\mathrm{div})$-convergence has been proved in the same reference only for asymptotically parallelogram meshes (i.e., meshes built on shape-regular quadrilaterals such that the distance of the vertices of each element to those of a parallelogram are higher order terms). To the best of the authors knowledge, a similar analysis has not been made on general hexahedra 3D meshes.

On the other hand, Arnold et al. [1,3] have recently noted, for various types of 2D finite elements, that the extension to general quadrilaterals of approximation results valid for rectangular elements is less straightforward than what it was typically thought. Moreover, they have shown that the order of convergence can deteriorate when some non-standard finite elements are used in distorted quadrilaterals, even though they are shape-regular. The same authors have considered in [2] the approximation properties of 2D quadrilateral finite element spaces of 
vector fields defined by the Piola transform, as it is the case of Raviart-Thomas elements. For these elements, they have shown degradation of the convergence order for some trapezoid meshes as compared to rectangular ones.

We show in this paper that $\mathrm{H}(\mathrm{div})$-convergence is preserved on shape-regular asymptotically parallelepiped 3D meshes and we demonstrate the need of this assumption by means of a numerical example.

\section{The lowest-order Raviart-Thomas hexahedron}

A hexahedron is the image of an invertible trilinear map defined on a reference element: the unit cube $\widehat{K}:=$ $[0,1]^{3}$. (Throughout the paper, we use "hatted" variables to denote magnitudes in the reference element.) Indeed, let $\boldsymbol{F}_{K}: \widehat{K} \rightarrow \mathbb{R}^{3}$ be a trilinear one-to-one map with a strictly positive Jacobian: $J_{K}(\widehat{\boldsymbol{x}}):=\operatorname{det}\left[\widehat{D} \boldsymbol{F}_{K}(\widehat{\boldsymbol{x}})\right]>0$ $\forall \widehat{\boldsymbol{x}} \in \widehat{K}$, where $\widehat{D} \boldsymbol{F}_{K}$ denotes the Jacobian matrix of the mapping $\boldsymbol{F}_{K}$. The corresponding hexahedron is $K:=$ $\boldsymbol{F}_{K}(\widehat{K})$. Notice that $K$ does not need to have planar faces; the edges are always straight segments, but each face is planar if and only if its four vertices lie on a plane.

Let $h_{K}$ be the diameter of $K$. Let $\bar{\sigma}_{K}$ and $\underline{\sigma}_{K}$ be two strictly positive constants such that

$$
h_{K}^{-1}\left|\widehat{D} \boldsymbol{F}_{K}(\widehat{\boldsymbol{x}})\right| \leq \bar{\sigma}_{K} \quad \forall \widehat{\boldsymbol{x}} \in \widehat{K} \quad \text { and } \quad h_{K}^{-1}\left|D\left(\boldsymbol{F}_{K}^{-1}\right)(\boldsymbol{x})\right|^{-1} \geq \underline{\sigma}_{K} \quad \forall \boldsymbol{x} \in K .
$$

Here and thereafter, $|\cdot|$ denotes the standard Euclidean norm in $\mathbb{R}^{3}$ as well as the corresponding subordinated matrix norm in $\mathbb{R}^{3 \times 3}$. Notice that constants $\bar{\sigma}_{K}$ and $\underline{\sigma}_{K}$ measure the shape regularity of the hexahedron.

We recall that the cofactor matrix of $\widehat{D} \boldsymbol{F}_{K}(\widehat{\boldsymbol{x}})$ satisfies $\operatorname{Cof}\left[\widehat{D} \boldsymbol{F}_{K}(\widehat{\boldsymbol{x}})\right]^{\mathrm{t}}=J_{K}(\widehat{\boldsymbol{x}})\left[\widehat{D} \boldsymbol{F}_{K}(\widehat{\boldsymbol{x}})\right]^{-1}$. Explicit computations lead to the following bounds:

$$
\underline{\sigma}_{K}^{3} h_{K}^{3} \leq J_{K}(\widehat{\boldsymbol{x}}) \leq \bar{\sigma}_{K}^{3} h_{K}^{3} \quad \text { and } \quad \underline{\sigma}_{K}^{3} \bar{\sigma}_{K}^{-1} h_{K}^{2} \leq\left|\operatorname{Cof}\left(\widehat{D} \boldsymbol{F}_{K}\right)(\widehat{\boldsymbol{x}})\right| \leq \bar{\sigma}_{K}^{3} \underline{\sigma}_{K}^{-1} h_{K}^{2} \quad \forall \widehat{\boldsymbol{x}} \in \widehat{K} .
$$

To measure the "deviation" of a hexahedron $K$ with respect to a parallelepiped, we decompose $\boldsymbol{F}_{K}$ as a sum of two parts, one affine and the other depending on four geometrical vector parameters, $\varepsilon_{1}, \varepsilon_{2}, \varepsilon_{3}, \varepsilon_{4} \in \mathbb{R}^{3}$ (see Fig. 1). Indeed, let $\boldsymbol{\alpha}_{i}:=\boldsymbol{F}_{K}\left(\boldsymbol{e}_{i}\right) \in \mathbb{R}^{3}$, with $\boldsymbol{e}_{i}$ being the $i^{\text {th }}$ canonical vector, $i=1,2,3$. For $\widehat{\boldsymbol{x}}=\left(\widehat{x}_{1}, \widehat{x}_{2}, \widehat{x}_{3}\right)$, let $\boldsymbol{F}_{K}(\widehat{\boldsymbol{x}})=\boldsymbol{F}_{K}^{\mathrm{l}}(\widehat{\boldsymbol{x}})+\boldsymbol{F}_{K}^{\mathrm{d}}(\widehat{\boldsymbol{x}})$, with

$\boldsymbol{F}_{K}^{\mathrm{l}}(\widehat{\boldsymbol{x}})=\widehat{x}_{1} \boldsymbol{\alpha}_{1}+\widehat{x}_{2} \boldsymbol{\alpha}_{2}+\widehat{x}_{3} \boldsymbol{\alpha}_{3} \quad$ and $\quad \boldsymbol{F}_{K}^{\mathrm{d}}(\widehat{\boldsymbol{x}})=\widehat{x}_{2} \widehat{x}_{3} \varepsilon_{1}+\widehat{x}_{1} \widehat{x}_{3} \varepsilon_{2}+\widehat{x}_{1} \widehat{x}_{2} \varepsilon_{3}+\widehat{x}_{1} \widehat{x}_{2} \widehat{x}_{3}\left(\varepsilon_{4}-\varepsilon_{1}-\varepsilon_{2}-\varepsilon_{3}\right)$, where $\boldsymbol{F}_{K}^{\mathrm{l}}$ is the linear part and $\boldsymbol{F}_{K}^{\mathrm{d}}$ the "deviation" (for simplicity, we have not considered translations). Let us recall that if the faces of the element $K$ are planar, then $\varepsilon_{4}=\varepsilon_{1}+\varepsilon_{2}+\varepsilon_{3}$ and the cubic term of $\boldsymbol{F}_{K}^{\mathrm{d}}$ vanishes.
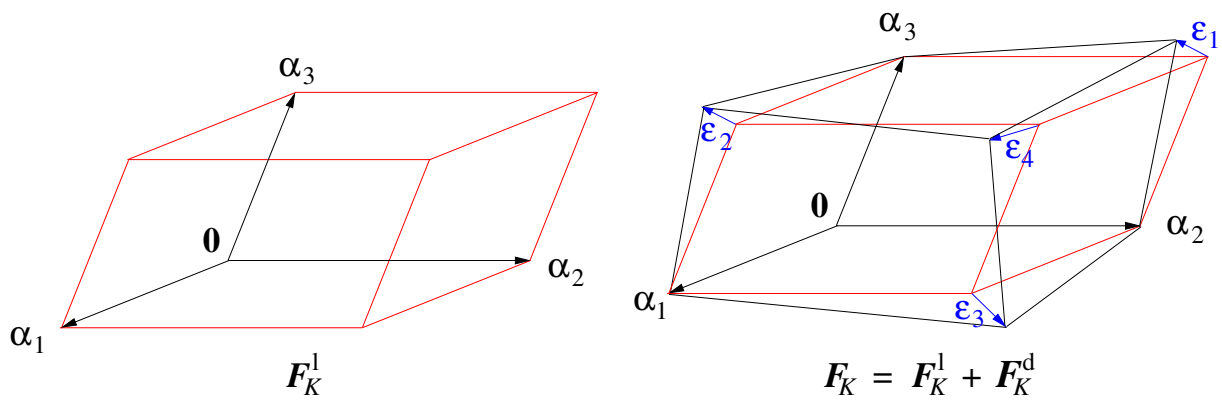

Figure 1. Image of $\widehat{K}$ by $\boldsymbol{F}_{K}^{1}$ (left) and $\boldsymbol{F}_{K}$ decomposed as $\boldsymbol{F}_{K}^{1}+\boldsymbol{F}_{K}^{\mathrm{d}}$ (right).

Let $\varepsilon_{K}:=\max _{1 \leq i \leq 4}\left|\varepsilon_{i}\right|$. The following lemma has been proved in [6]. Here and thereafter, $C$ denotes a generic strictly positive constant not necessarily the same at each occurrence.

Lemma 2.1 There exists a strictly positive constant $C$ such that, for any hexahedron $K$,

$$
\max _{\widehat{\boldsymbol{x}} \in \widehat{K}}\left|\widehat{\nabla} J_{K}(\widehat{\boldsymbol{x}})\right| \leq C \varepsilon_{K} h_{K}^{2} \quad \text { and } \quad \max _{\widehat{\boldsymbol{x}} \in \widehat{K}}\left|\frac{\partial}{\partial \widehat{x}_{k}}\left(\operatorname{Cof}\left[\widehat{D} \boldsymbol{F}_{K}(\widehat{\boldsymbol{x}})\right]\right)_{i j}\right| \leq C \varepsilon_{K} h_{K}, \quad 1 \leq i, j, k \leq 3 .
$$

Remark 1 Similar estimates were proved by Thomas [15] in 2D. In this case there is a unique vector parameter $\varepsilon \in \mathbb{R}^{2}$ which measures the deviation of a quadrilateral $K$ with respect to a parallelogram. Actually, Thomas used another one: the vector $\boldsymbol{\delta}$ joining the midpoints of the diagonals of $K$. However, it is simple to show that $\boldsymbol{\delta}=\frac{1}{2} \varepsilon$. 
Given a vector field $\boldsymbol{u}$ defined on $K$, Raviart-Thomas elements discretize the whole vector field instead of its components separately. The degrees of freedom of the lowest-order Raviart-Thomas hexahedron are the mean values of the fluxes of $\boldsymbol{u}$ on each face of the hexahedron. To define these elements, we change coordinates to the reference unit cube and use the Piola transform associated with this change of coordinates (see for instance [11]):

$$
\widehat{\boldsymbol{u}}(\widehat{\boldsymbol{x}}):=J_{K}(\widehat{\boldsymbol{x}})\left[\widehat{D} \boldsymbol{F}_{K}(\widehat{\boldsymbol{x}})\right]^{-1} \boldsymbol{u}\left(\boldsymbol{F}_{K}(\widehat{\boldsymbol{x}})\right), \quad \widehat{\boldsymbol{x}} \in \widehat{K} .
$$

A lowest-order Raviart-Thomas element on $K$ is a vector field $\boldsymbol{u}$ whose Piola transform $\widehat{\boldsymbol{u}}$ satisfies

$$
\widehat{\boldsymbol{u}} \in \widehat{\mathcal{R}}:=\mathcal{P}_{1,0,0}(\widehat{K}) \times \mathcal{P}_{0,1,0}(\widehat{K}) \times \mathcal{P}_{0,0,1}(\widehat{K}),
$$

where $\mathcal{P}_{i, j, k}(\widehat{K})$ denotes the set of polynomial functions defined on $\widehat{K}$ of degrees $i, j$, and $k$ in $\widehat{x}_{1}, \widehat{x}_{2}$, and $\widehat{x}_{3}$, respectively. These vector fields have constant normal components on each face of the reference unit cube $\widehat{K}$. Since these constant normal components determine a unique vector field of this type, they can be taken as the degrees of freedom defining this element. Thus, the space of lowest-order Raviart-Thomas finite elements on $K$ is

$$
\mathcal{R}_{K}:=\left\{\boldsymbol{u}: K \rightarrow \mathbb{R}^{3}: \widehat{\boldsymbol{u}} \in \widehat{\mathcal{R}}\right\}
$$

\section{Approximation properties}

Given $\boldsymbol{u} \in \mathrm{H}^{r}(K)^{3}, r>\frac{1}{2}$, the Raviart-Thomas interpolant of $\boldsymbol{u}$ in $K$ is defined as the unique $\boldsymbol{u}_{K} \in \mathcal{R}_{K}$ such that $\int_{F} \boldsymbol{u}_{K} \cdot \boldsymbol{n}_{F}=\int_{F} \boldsymbol{u} \cdot \boldsymbol{n}_{F}$ for all faces $F$ of $K\left(\boldsymbol{n}_{F}\right.$ denotes a unit vector normal to $\left.F\right)$. The mapping $\boldsymbol{u} \mapsto \boldsymbol{u}_{K}$ extends by duality arguments to $\boldsymbol{u} \in \mathrm{H}(\operatorname{div}, K) \cap \mathrm{H}^{r}(K)^{3} \forall r>0$, defining a bounded linear operator (see [11]).

Error estimates for this interpolation in 2D were proved in [15] for $\boldsymbol{u} \in \mathrm{H}^{1}(K)^{3}$ with div $\boldsymbol{u} \in \mathrm{H}^{1}(K)$. The arguments in this reference have been adapted in [6] to 3D and fractional Sobolev spaces yielding the following result:

Theorem 3.1 If $\boldsymbol{u} \in \mathrm{H}(\operatorname{div}, K) \cap \mathrm{H}^{r}(K)^{3}, 0<r \leq 1$, then $\exists C>0$, only depending on $\bar{\sigma}_{K}$, $\underline{\sigma}_{K}$, and $r$, such that

$$
\left\|\boldsymbol{u}-\boldsymbol{u}_{K}\right\|_{0, K} \leq C\left(\varepsilon_{K} h_{K}^{-1}\|\boldsymbol{u}\|_{0, K}+h_{K}^{r}|\boldsymbol{u}|_{r, K}+h_{K}\|\operatorname{div} \boldsymbol{u}\|_{0, K}\right),
$$

If moreover $\operatorname{div} \boldsymbol{u} \in \mathrm{H}^{s}(K), 0<s \leq 1$, then $\exists C>0$, only depending on $\bar{\sigma}_{K}, \underline{\sigma}_{K}$, and s, such that

$$
\left\|\operatorname{div}\left(\boldsymbol{u}-\boldsymbol{u}_{K}\right)\right\|_{0, K} \leq C\left(\varepsilon_{K} h_{K}^{-1}\|\operatorname{div} \boldsymbol{u}\|_{0, K}+h_{K}^{s}|\operatorname{div} \boldsymbol{u}|_{s, K}\right) .
$$

Remark 2 If $\boldsymbol{u} \in \mathrm{H}^{r}(K)^{3}$ with $r>\frac{1}{2}$, then the first estimate above can be improved as follows:

$$
\left\|\boldsymbol{u}-\boldsymbol{u}_{K}\right\|_{0, K} \leq C\left(\varepsilon_{K} h_{K}^{-1}\|\boldsymbol{u}\|_{0, K}+h_{K}^{r}|\boldsymbol{u}|_{r, K}\right) .
$$

Remark 3 Girault and Raviart ([12], Theorem III.4.4) have extended some arguments from Thomas ([15], Theorem III.4.3) to prove the following improved estimate in $2 D$ for $\boldsymbol{u} \in \mathrm{H}^{1}(K)^{3}$ :

$$
\left\|\boldsymbol{u}-\boldsymbol{u}_{K}\right\|_{0, K} \leq C h_{K}|\boldsymbol{u}|_{1, K} .
$$

More recently, Arnold et al. ([2], Theorem 7) have obtained a similar result valid for 2D Raviart-Thomas elements of arbitrary order. However, the arguments of these references do not seem to hold in $3 D$. Indeed, both rely on the fact that, in $2 D$, the columns of the matrix $\operatorname{Cof}\left(\widehat{D} \boldsymbol{F}_{K}\right)^{\mathrm{t}}$ belong to $\mathcal{P}_{1,0} \times \mathcal{P}_{0,1}$, which, in this case, corresponds to $\widehat{\mathcal{R}}$ (see [2], Lemma 9). Instead, in 3D, these columns belong to $\mathcal{P}_{2,1,1} \times \mathcal{P}_{1,2,1} \times \mathcal{P}_{1,1,2} \neq \widehat{\mathcal{R}}$.

Next, consider a family of partitions in hexahedra $\left\{\mathcal{T}_{h}\right\}$ of a given polyhedral bounded domain $\Omega \subset \mathbb{R}^{3}$; as usual, $h:=\max _{K \in \mathcal{T}_{h}} h_{K}$ denotes the mesh-size. We recall that the family is regular if $\exists \sigma_{1}, \sigma_{2}>0$, the same for all meshes, such that

$$
\bar{\sigma}_{K} \leq \sigma_{1} \quad \text { and } \quad \underline{\sigma}_{K} \geq \sigma_{2} \quad \forall K \in \mathcal{T}_{h} .
$$

On the other hand, $\left\{\mathcal{T}_{h}\right\}$ is a family of asymptotically parallelepiped meshes, if for each element $K \in \mathcal{T}_{h}$ there exists a parallelepiped such that the distances of the vertices of $K$ to those of the parallelepiped are $\mathcal{O}\left(h_{K}^{2}\right)$. More precisely, if there exists a strictly positive constant $\sigma_{3}$, the same for all meshes, such that

$$
\varepsilon_{K} \leq \sigma_{3} h_{K}^{2} \quad \forall K \in \mathcal{T}_{h} .
$$

Notice that, as a consequence of Lemma 2.1, if $\left\{\mathcal{T}_{h}\right\}$ is a family of asymptotically parallelepiped meshes, then the estimates for the derivatives of $J_{K}$ and the entries of Cof $\left(\widehat{D} \boldsymbol{F}_{K}\right)$ are one order larger than those given by (1) for $J_{K}$ and $\left|\operatorname{Cof}\left(\widehat{D} \boldsymbol{F}_{K}\right)\right|$, respectively. 
Raviart-Thomas elements are $\mathrm{H}(\operatorname{div})$-conforming in the following sense: Given $\boldsymbol{u} \in \mathrm{H}(\operatorname{div}, \Omega) \cap \mathrm{H}^{r}(\Omega)^{3}$ with $r>0$ (or $\boldsymbol{u} \in \mathrm{H}^{r}(\Omega)^{3}$ with $r>\frac{1}{2}$ ), let $\boldsymbol{u}^{\mathrm{R}} \in \mathrm{L}^{2}(\Omega)^{3}$ be defined by $\left.\boldsymbol{u}^{\mathrm{R}}\right|_{K}:=\boldsymbol{u}_{K} \forall K \in \mathcal{T}_{h}$; then $\boldsymbol{u}^{\mathrm{R}} \in \mathrm{H}(\operatorname{div}, \Omega$ ). Consequently, $\boldsymbol{u}^{\mathrm{R}}$, which is called the Raviart-Thomas interpolant of $\boldsymbol{u}$ in $\Omega$, belongs to

$$
\mathcal{R}_{h}:=\left\{\boldsymbol{u}_{h} \in \mathrm{H}(\operatorname{div}, \Omega):\left.\boldsymbol{u}_{h}\right|_{K} \in \mathcal{R}_{K} \forall K \in \mathcal{T}_{h}\right\} .
$$

The following result is an immediate consequence of Theorem 3.1:

Corollary 3.2 Let $\boldsymbol{u} \in \mathrm{H}^{r}(\Omega)^{3}$ with $0<r \leq 1$ be such that $\operatorname{div} \boldsymbol{u} \in \mathrm{H}^{s}(\Omega)$ with $0<s \leq 1$. Let $\boldsymbol{u}^{\mathrm{R}} \in \mathcal{R}_{h}$ be the Raviart-Thomas interpolant of $\boldsymbol{u}$. If $\left\{\mathcal{T}_{h}\right\}$ is a regular family of asymptotically parallelepiped meshes of $\Omega$, then

$$
\left\|\boldsymbol{u}-\boldsymbol{u}^{\mathrm{R}}\right\|_{0, \Omega} \leq C h^{r}\left(\|\boldsymbol{u}\|_{r, \Omega}+\|\operatorname{div} \boldsymbol{u}\|_{0, \Omega}\right) \quad \text { and } \quad\left\|\operatorname{div}\left(\boldsymbol{u}-\boldsymbol{u}^{\mathrm{R}}\right)\right\|_{0, \Omega} \leq C h^{s}\|\operatorname{div} \boldsymbol{u}\|_{s, \Omega} .
$$

Remark 4 The power 2 of $h_{K}$ in the definition of asymptotically parallelepiped meshes (2) is somewhat arbitrary. Indeed, the corollary above holds true as far as $\varepsilon_{K} \leq C h_{K}^{1+\max \{r, s\}}$.

\section{Need of the asymptotically parallelepiped assumption on the meshes}

Hexahedral Raviart-Thomas elements have been successfully used to numerically solve the following spectral problem, which models the acoustic vibrations in a rigid cavity:

Find $\lambda \in \mathbb{R}$ and $\boldsymbol{u} \in \mathrm{H}_{0}(\operatorname{div}, \Omega), \boldsymbol{u} \neq \mathbf{0}$, such that

$$
\int_{\Omega} \operatorname{div} \boldsymbol{u} \operatorname{div} \boldsymbol{v}=\lambda \int_{\Omega} \boldsymbol{u} \cdot \boldsymbol{v} \quad \forall \boldsymbol{v} \in \mathrm{H}_{0}(\operatorname{div}, \Omega) .
$$

We recall that $\mathrm{H}_{0}(\operatorname{div}, \Omega):=\{\boldsymbol{v} \in \mathrm{H}(\operatorname{div}, \Omega): \boldsymbol{v} \cdot \boldsymbol{n}=0$ on $\partial \Omega\}$, where $\boldsymbol{n}$ is the outer unit vector normal to $\partial \Omega$.

The following discrete problem is obtained by using lowest-order Raviart-Thomas hexahedral elements:

Find $\lambda_{h} \in \mathbb{R}$ and $\boldsymbol{u}_{h} \in \mathcal{R}_{h}^{0}, \boldsymbol{u}_{h} \neq \mathbf{0}$, such that

$$
\int_{\Omega} \operatorname{div} \boldsymbol{u}_{h} \operatorname{div} \boldsymbol{v}_{h}=\lambda_{h} \int_{\Omega} \boldsymbol{u}_{h} \cdot \boldsymbol{v}_{h} \quad \forall \boldsymbol{v}_{h} \in \mathcal{R}_{h}^{0} .
$$

In the expression above, $\mathcal{R}_{h}^{0}:=\mathcal{R}_{h} \cap \mathrm{H}_{0}(\operatorname{div}, \Omega)=\left\{\boldsymbol{v}_{h} \in \mathcal{R}_{h}: \boldsymbol{v}_{h} \cdot \boldsymbol{n}=0\right.$ on $\left.\partial \Omega\right\}$.

For a family of meshes such that Corollary 3.2 holds true, the abstract spectral theory (see [4]) has been applied in [6] to prove convergence for eigenvalues and eigenfunctions. The asymptotically parallelepiped assumption is essential for this convergence to hold, as we show in the following example.

Consider a 2D cavity $\Omega:=[0,1]^{2}$. The eigenvalues of (3) are analytically known:

$$
\lambda_{m n}=\pi^{2}\left(m^{2}+n^{2}\right), \quad m, n=0,1,2, \ldots
$$

To test the method with a family of non-asymptotically parallelogram meshes, we have used partitions of the domain into $N \times N$ congruent trapezoids, all similar to the trapezoid with vertices $(0,0),\left(\frac{1}{2}, 0\right),\left(\frac{1}{2}, \frac{2}{3}\right)$ and $\left(0, \frac{1}{3}\right)$, as shown in Fig. 2. Clearly, the elements of these meshes are uniformly far from being parallelograms. They were used in $[1,2,3]$ to show that the order of convergence of some quadrilateral finite elements deteriorates, even though shape-regular meshes are used. We have solved the problem with uniform meshes of $N \times N$ squares, as well.
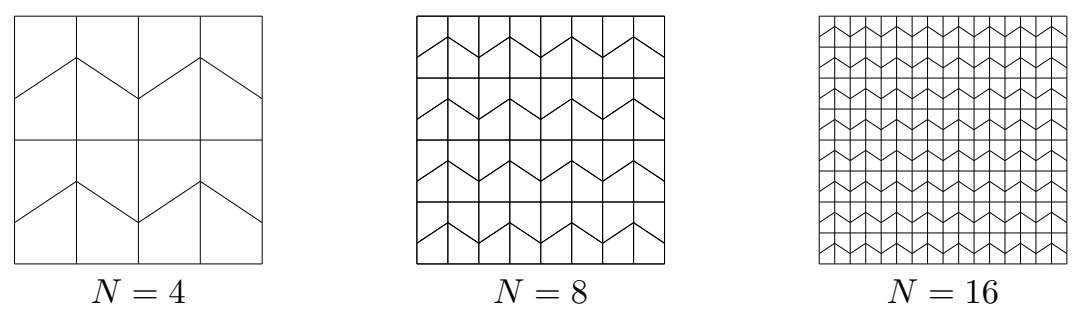

Figure 2. Non-asymptotically parallelogram trapezoid meshes in $\Omega=[0,1]^{2}$.

Tables 1 and 2 show the five smallest eigenvalues $\lambda_{m n}$ computed with these square and trapezoid meshes, respectively. Each table includes the approximations of the eigenvalues obtained by extrapolating the computed ones, that we denote $\widehat{\lambda}_{m n}$ and $\widetilde{\lambda}_{m n}$, respectively, as well as the computed orders of convergence. Table 1 also includes the exact eigenvalues and Table 2 the ratios between the extrapolated values obtained with each method. 
Table 1

Eigenvalues computed with square Raviart-Thomas elements.

\begin{tabular}{cccccccc}
\hline & $N=8$ & $N=16$ & $N=32$ & $N=64$ & $\widehat{\lambda}_{m n}$ & Order & Exact \\
\hline$\lambda_{10}$ & 9.997081 & 9.901356 & 9.877535 & 9.871588 & 9.869585 & 2.00 & 9.869604 \\
$\lambda_{01}$ & 9.997081 & 9.901356 & 9.877535 & 9.871588 & 9.869585 & 2.00 & 9.869604 \\
$\lambda_{11}$ & 19.994162 & 19.802705 & 19.755066 & 19.743170 & 19.739168 & 2.00 & 19.739209 \\
$\lambda_{20}$ & 41.546571 & 39.988324 & 39.605414 & 39.510139 & 39.479419 & 2.01 & 39.478418 \\
$\lambda_{02}$ & 41.546571 & 39.988324 & 39.605414 & 39.510139 & 39.479419 & 2.01 & 39.478418 \\
\hline
\end{tabular}

Table 2

Eigenvalues computed with trapezoid Raviart-Thomas elements.

\begin{tabular}{cccccccc}
\hline & $N=8$ & $N=16$ & $N=32$ & $N=64$ & $\widetilde{\lambda}_{m n}$ & Order & $\widetilde{\lambda}_{m n} / \widehat{\lambda}_{m n}$ \\
\hline$\lambda_{10}$ & 10.347442 & 10.282911 & 10.266965 & 10.262896 & 10.261610 & 2.01 & 1.039721 \\
$\lambda_{01}$ & 10.394167 & 10.294620 & 10.269891 & 10.263626 & 10.261597 & 2.00 & 1.039719 \\
$\lambda_{11}$ & 20.736680 & 20.576279 & 20.536520 & 20.526438 & 20.523194 & 2.00 & 1.039719 \\
$\lambda_{20}$ & 42.451339 & 41.390093 & 41.131825 & 41.067538 & 41.047158 & 2.02 & 1.039710 \\
$\lambda_{02}$ & 43.196809 & 41.576587 & 41.178634 & 41.079252 & 41.046629 & 2.01 & 1.039697 \\
\hline
\end{tabular}

We notice that the method converges in both cases with an almost perfect quadratic order. However, in the case of the trapezoid elements, it converges to wrong eigenvalues! This can only happen if $\left\|\boldsymbol{u}-\boldsymbol{u}^{\mathrm{R}}\right\|_{\mathrm{H}(\mathrm{div}, \Omega)}$ does not converge to zero as $h$ goes to zero. Thus, this numerical test shows that such convergence fails for these particular non-asymptotically parallelepiped meshes. A more detailed analysis of this phenomenon can be found in [6], including the reason why the quotients between exact and wrong eigenvalues are almost constant.

\section{Acknowledgments}

First author partially supported by MCYT (Spain) Project DPI2001-1613-C02-02 and by Xunta de Galicia (Spain) Project PGIDIT02PXIC2070IPN. Second author partially supported by MCYT (Spain) Projects DPI20011613-C02-02 and BFM2001-3261-C02-02. Third author partially supported by MCYT (Spain) Project VEM200320069-C03-02. Fourth author partially supported by FONDAP in Applied Mathematics (Chile). This paper was done during a sabbatical stay of the fourth author at Universidade de Santiago de Compostela, supported by Secretaría de Estado de Educación y Universidades through the grant SAB2003-0215.

\section{References}

[1] D.N. Arnold, D. Boffi, R.S. Falk, Approximation by quadrilateral finite elements, Math. Comp. 71 (2002) 909-922.

[2] D.N. Arnold, D. Boffi, R.S. Falk, Quadrilateral H(div) finite elements, SIAM J. Numer. Anal. (to appear).

[3] D.N. Arnold, D. Boffi, R.S. Falk, L. Gastaldi, Finite element approximation on quadrilateral meshes, Comm. Numer. Methods Engrg. 17 (2001) 805-812.

[4] I. Babuška, J. Osborn, Eigenvalue problems, Handbook of Numerical Analysis, Vol. II, P.G. Ciarlet, P.L. Lions, eds. North-Holland, Amsterdam, 1991, pp. 641-787.

[5] A. Bermúdez, R. Durán, M.A. Muschietti, R. Rodríguez, J. Solomin, Finite element vibration analysis of fluid-solid systems without spurious modes, SIAM J. Numer. Anal. 32 (1995) 1280-1295.

[6] A. Bermúdez, P. Gamallo, M.R. Nogueiras, R. Rodríguez, Approximation of a structural acoustic vibration problem by hexahedral finite elements (submitted).

[7] A. Bermúdez, P. Gamallo, R. Rodríguez, A hexahedral face element for elastoacoustic vibration problems, J. Acoustical Soc. Amer. 109 (2001) 422-425.

[8] A. Bermúdez, P. Gamallo, R. Rodríguez, An hexahedral face element method for the displacement formulation of structural acoustics problems, J. Comput. Acoust. 9 (2001) 911-918.

[9] A. Bermúdez, L. Hervella-Nieto, R. Rodríguez, Finite element computation of three dimensional elastoacoustic vibrations, J. Sound \& Vibration, 219 (1999) 277-304.

[10] A. Bermúdez, R. Rodríguez, Finite element computation of the vibration modes of a fluid-solid system, Comput. Methods Appl. Mech. Engrg. 119 (1994) 355-370.

[11] F. Brezzi, M. Fortin, Mixed and Hybrid Finite Element Methods, Springer, New York, 1991.

[12] V. Girault, P.A. Raviart, Finite Element Methods for Navier-Stokes Equations. Theory and Algorithms, Springer-Verlag, Berlin, 1986

[13] J.C. Nédélec, Mixed finite elements in $\mathbb{R}^{3}$, Numer. Math. 35 (1980) 315-341.

[14] P.A. Raviart, J.M. Thomas, A mixed finite element method for second order elliptic problems, Mathematical Aspects of Finite Element Methods, I. Galligani, E. Magenes, eds. Lecture Notes in Mathematics, 606, Springer-Verlag, Berlin, 1977, pp. $292-315$.

[15] J.M. Thomas, Sur l'Analyse Numérique des Méthodes d'Éléments Finis Hybrides et Mixtes, Thèse de Doctorat d'Etat, Université Pierre et Marie Curie, Paris, 1977. 